We appreciate the response to this publication feature and welcome all contributions. Contributions may be sent to José A. Mascorro, our Technical Editor, at his e-mail address: jmascor@tulane.edu. José may also be reached at the Department of Structural and Cellular Biology,

Tulane University Health Sciences Center, 1430 Tulane Ave., New Orleans, LA 70112 and Ph: (504) 584-2747 Fax:

\section{Making Your own Molds for the EM lab}

\section{Mary Mager}

University of British Columbia

mager@interchange.ubc.ca

Silicone rubber can be cast into many useful shapes quite easily in the laboratory. I make my own metallurgical mounting molds for mounting samples in epoxy resin. I have patterns for making molds which are one inch in diameter by $5 / 8$ inch high and others for making 1.25 inch and 0.75 inch diameter molds. They are all based on the disposable Tri-pour plastic beakers. You can use dixie cups or other disposable containers and you can make almost any size and shape molds that you can design a suitable reverse mold for.

For the one inch molds, I use a $50 \mathrm{ml}$. beaker which is about 1.5 inches in diameter at the bottom. There is a hole in the center of the bottom of the beaker to admit a small screw that is about 0.25 inches long. For the hollow in the mold, I have an aluminum cylinder, one inch in diameter and $5 / 8$ inch tall. The bottom of the cylinder is machined a little concave to match the shape of the bottom of the

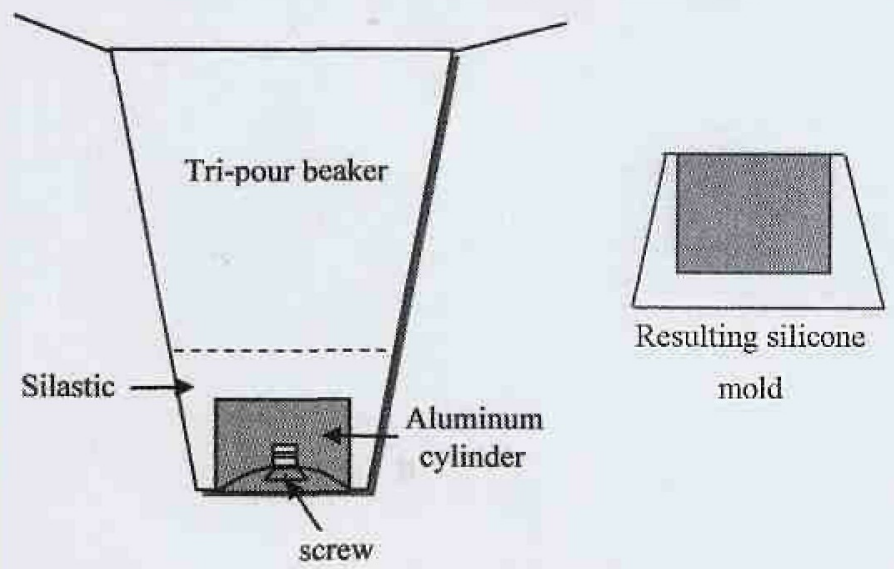

beaker, and tapped with a hole to receive the small screw. This allows you to screw the aluminum cylinder tightly down to the bottom of the beaker to prevent the silicone rubber from running under it.

The Silastic E RTV Silicone rubber kit (Dow Corning Corp.) is mixed according to directions. I use a 110 gram mixture to make five molds at once. The rubber base is weighed, then the catalyst is weighed, added and mixed thoroughly in a disposable $200 \mathrm{ml}$ cup. The mixture is then out-gassed in a vacuum desiccator to remove as much of the air trapped in the Silastic as possible. The mixture is dispensed into the Tri-pour beaker reverse mold, until there is at least $3 / 8$ inch of Silastic over the aluminum cylinder. Dispense slowly so that the Silastic has time to run down into the corners and minimum air is trapped. The individual beakers and the original Silastic mix are both out-gassed in a vacuum dessicator.

The Silastic will cure at room temperature over 24 hours. The serew is removed and the aluminum cylinder pushed up to remove the rubber mold from the beaker. It may help to run a thin spatula around the inside of the beaker to release the sticky rubber. Any flashing or rubber that has run under the cylinder can be trimmed with a sharp blade. I find it helps to spray the rubber molds lightly with a silicone mold release spray each time before pouring in the epoxy resin, when using the silicon rubber molds to mount samples. The molds will dry out and crack in use, but I use mine for six months to a year before replacing them.

The chemicals in the Silastic E RTV Silicone Rubber Kit can be irritant and toxic. Read all the MSDS and warnings and wear proper protective gear when mixing and dispensing the silicone rubber.

\section{Vascular Corrosion Casting}

Fred E. Hossler, East Tennessee State University J.H. Quillen College of Medicine hossler@mail.etsu.edu

With the development of low viscosity resins, casts of the fine capillary beds of tissues and organs were attainable, and these could be viewed and studied in detail in the scanning electron microscope $[1,2]$. The advantage of such casts is that they permitted for the first time, viewing of the intact, three-dimensional complex vasculature of tissues and organs. It follows that once vascular casts are obtained, quantitative measurements of various types could also be made from the casts if care was exercised. Of course, in theory, it should be possible to prepare casts of the three-dimensional lumenal space of any structure, biological or other [3]. This report will concentrate only on applications of corrosion casting to the vasculature of animal species.

Several modifications of the general method of preparing vascular corrosion casts are currently used in different laboratories, as well as several types of resins [4]. Outlined here is one method. After appropriate anesthetic and anticoagulant are administered (for rat: ip injections of nembutal, $60 \mathrm{mg} / \mathrm{kg}$; heparin, $700 \mathrm{U} / \mathrm{kg}$ ), an artery leading to the organ of interest is located and cannulated. The cannula is attached to one port on a three-way valve [5]. Another port on the valve is attached to a saline or Ringer=s reservoir (warmed to $37^{\circ} \mathrm{C}$ ), and the third port on the valve is attached to a syringe barrel. The entire system is joined to a manometer and pressure bulb for controlling perfusion pressure. A vein draining the organ of interest is opened as an outlet, and blood is flushed from the organ with the warm saline or Ringers=s solution at a pressure appropriate for the animal $(80-100 \mathrm{~mm} \mathrm{Hg}$ for rats). Resin $(8 \mathrm{ml}$ Mercox, 2 $\mathrm{ml}$ methylmethacrylate monomer, and $0.3 \mathrm{ml}$ catalyst; prepared just before use) is placed in the syringe barrel and infused through the same cannula until the onset of polymerization (about 8-10 min). The

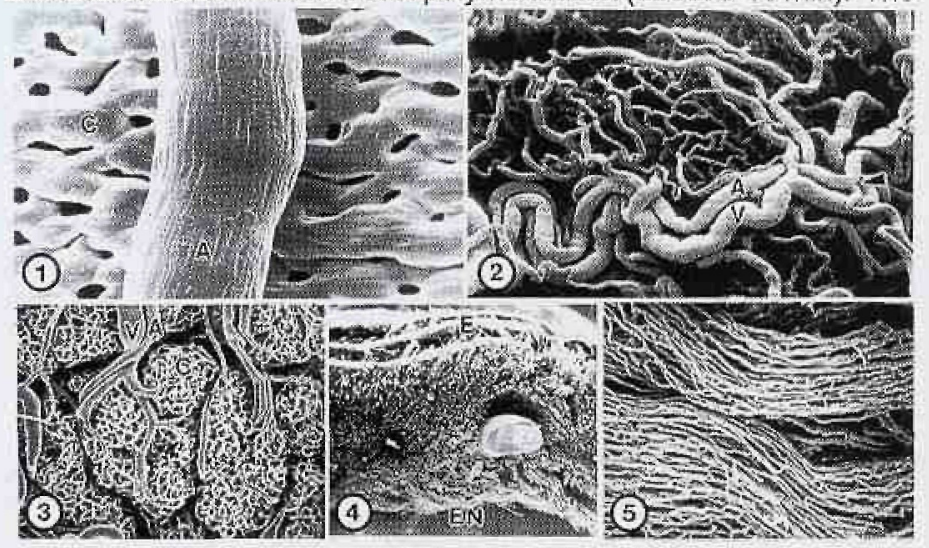

Figure 1. Vascular corrosion cast of frog retina. $A$, artery; $C$ capillary. $\times 320$

Figure 2. Vascular corrosion cast of rabbit bladder. A, artery; $V$, vein: arrowhead, sphincter. $\times 132$

Figure 3. Vascular cast of avian salt gland. A, artery; $V$, vein; $C$, capillary bed. $\times 106$

Figure 4. Vascular cast of right ventricular free wall of rat heart. $E$, epicardium; $E N$, endocardium. $\times 34$

Figure 5. Vascular cast of capillary beds of trabecular muscle of rat heart. $\times 121$ 
perfused tissue is immersed in hot water for at least several hours to complete polymerization, the tissue is removed with alternating treatments of $5 \% \mathrm{KOH}$ and distilled water, and the resulting cast is cleaned with $5 \%$ formic acid ( $15 \mathrm{~min}$ ) and distilled water. The cast is dried by lyophilization, mounted on a stub, and sputter coated for routine SEM observation.

Example corrosion casts from various tissues including heart [6], lung [7], urinary bladder [8], and salt gland [9] are shown. Some of the types of anatomical information that can be obtained from casts include: distribution and 3-D anatomy of the microvasculature of tissues, venous valve structure, location of arterial sphincters, distribution and size of nuclear imprints, and some simple quantitative measurements, including vascular volume and vessel dimensions. Accurate, fine detailed measurements of vascular casts also are possible using 3-D morphometry techniques, scanning electron microscopy of stereo pairs, and digital image analysis [10]. Because of the natural fluorescence of the blue dye in the Mercox resin, corrosion casts also can be studied with confocal microscopy [11].

\section{References}

1. T. Murakami, Arch. Histol. Jpn. 32 (1971) 445.

2. J.A. Nowell, et al., Scanning Elect. Microsc., 1970 (1970) 249.

3. F.E Hossier, Microsc. Today, 98-7 (1998) 14.

4. A. Lametschwandtner, et al., Scanning Microsc., 4 (1990), 889.

5. K.R. Olson, J. Electron Microsc. Tech. 2 (1985) 217.

6. F.E. Hossler, et al., Scanning Elect. Microsc., 1986/IV (1986), 1469.

7. F.E. Hossler, et al, J. Electron Microsc. Tech. 19 (1991), 406.

8. F.E. Hossler and F. C. Monson, Anat, Rec. 243 (1995), 438.

9. F.E. Hossler and K. R. Olson, J. Exp. Zool. 254 (1990), 237.

10. B. Minnich, et al, J. Microsc. 195 (1999), 23.

11. K. Czymmek, et al., Microsc. Microanal. 6 (Suppl 2:Proc.)(2000), 562.

\section{Benzyldimethylamine (BDMA): Catalyst of Choice with Epoxy Embedding Media}

\author{
José A. Mascorro \\ Tulane University Health Sciences Center \\ jmascor@tulane.edu
}

The catalyst 2,4,6-Tri(dimethylaminomethyl)phenol (DMP-30) has been used to catalyze and polymerize epoxy resin embedding media since the earliest years of biological electron microscopy. Although DMP-30 works well, it is a highly viscous compound and media polymerized in this fashion likewise show a high viscous character. What follows is a rapid loss in fluidity in the medium, as well as shortened pot life, two factors that are absolutely essential in order to achieve complete specimen infiltration. Opting for less viscous catalysts, such as BDMA, presumably should yield lowered viscosity and prolonged fluidity in embedding media, thus ensuring complete infiltration and strengthened tissues subsequent to microtomy and ultrastructural study.

The differences between BDMA and DMP-30 become obvious following simple tests that define basic properties such as Average Flow Time (AFT: time necessary for known volume of catalyst to exit a viscosimeter), Volume Flow Rate (VFR: amount of catalyst flowing/time) and resulting Viscosity ( $\mathrm{CP}$; measure of fluidity and a correlate of AFTNFR). The tests are relatively simple and can be performed by filling a vertically-oriented viscosimeter with a known volume $(9 \mathrm{ml})$ of the catalyst and then recording the time necessary for the component to flow, under natural gravity, from the viscosimeter. It's possible to accurately predict (in advance) that if a chemical flows rapidly from the viscosimeter, it does so because the product is low in viscosity. On the other hand, if the product flows very slowly, it does so because the opposite is true. Applying these tests to BDMA and DMP-30 shows the following:

Table I

Characteristics of BDMA vs DMP-30

$\begin{array}{cccc} & \begin{array}{c}\text { Average } \\ \text { Flow Time }\end{array} & \begin{array}{c}\text { Volume } \\ \text { Flow Rate }\end{array} & \begin{array}{c}\text { Viscosity } \\ \text { (CP) }\end{array} \\ \text { BDMA } & 5.57 \text { sec/9ml } & 1.97 \mathrm{~m} / \mathrm{sec} & 0.84 \mathrm{cP} \\ \underline{\text { DMP-30 }} & \underline{2.11: 96 \mathrm{~min} / 9 \mathrm{ml}} & \underline{0.0682 \mathrm{~m} / \mathrm{sec}} & \underline{25.0 \mathrm{CP}}\end{array}$

These results clearly show that BDMA has a very rapid AFT, increased VFR, and thus is dramatically less viscous (@ $0.84 \mathrm{cP}$ ) than is DMP-30 (@25.0 cP). But do these interesting numbers by themselves suggest that utilizing BDMA, in lieu of DMP-30, would produce lowered viscosity and the attendant advantages? An LX 112-based embedding medium often preferred and well-known to this microscopist was prepared and catalyzed differently. The AFT, VFR, and resulting viscosity were tested immediately upon mixing and 60 minutes later.

Table II

LX 112/NSA/NMA catalyzed with BDMA or DMP-30

Time from

Initial mixing

@ 5 min with BDMA

(2) 5 min with DMP-30

@60 min with BDMA

@ 60 min with DMP-30

Hardening of any embedding medium naturally is accompanied by a loss of fluidity. These characteristics seem more prevalent when DMP-30 is the catalyst of choice. Immediately after mixing (5 minutes), DMP-30 causes a two-fold increase in viscosity as compared with BDMA, and this is accelerated even more after 60 minutes. It seems reasonable to presume that the medium with the lower viscosity, because it will remain fluid longer, will infiltrate tissues more completely. The information presented here also strongly suggests that the microscopist should not devout many hours to the process of infiltration, a common technique in many embedding protocols. This is not time efficient as it is clear that, once prepared, any combination of ingredients will undergo a two- and three-fold increase in viscosity and loss of fluidity within minutes of preparation. This is exactly what occurs when epoxy resins are placed in a mix with acid anhydrides and catalyzed with tertiary amines. Useful infiltration can only occur during the early pot life of the embedding medium. DMP-30 is of historical value and continues to be widely utilized today. BDMA, on the other hand, has always been recognized as a catalyst of choice by pioneer electron microscopists ${ }^{1}$

\section{References}

1. Audrey Glauert, personal conmmunication. XII ICEM. Seattle, 1990.

Acknowledgement:

Appreciation is extended to Dr. Gerald S. Kirby for assistance with viscosity measurements

\section{The Most Likely Sources of EDX} Copper Peaks in Samples Run by TEM

$$
\text { Paul Beauregard }
$$

Chemist and Electron Microscopist beaurega@westol.com

It is well known that TEM copper grids will give an EDX background of Cu peaks during EDX measurements. While there are a number of potential sources for these characteristic $\mathrm{Cu}$ peaks, the most intense source is likely the Cu grid itself. You can eliminate this source by using Be grids. Other potential sources can be addressed 